\title{
A Method for Determining the Mechanical Parameters of Solution Pore and Crevice Limestone Based on Porosity
}

\author{
Zheng Hu $\mathbb{D}$, ${ }^{1}$ Tao Wen $\mathbb{D}^{2,3}$ Kexun Zheng, ${ }^{2}$ and Yankun Wang $\mathbb{D}^{3}$ \\ ${ }^{1}$ Power China Guiyang Engineering Corporation, Ltd, Guiyang, Guizhou 550081, China \\ ${ }^{2}$ Hubei Cooperative Innovation Center of Unconventional Oil and Gas, Yangtze University, Wuhan, Hubei 430100, China
}

${ }^{3}$ School of Geosciences, Yangtze University, Wuhan, Hubei 430100, China

Correspondence should be addressed to Tao Wen; wentao200840@yangtzeu.edu.cn

Zheng $\mathrm{Hu}$ and Tao Wen contributed equally to this work.

Received 25 September 2020; Revised 15 January 2021; Accepted 22 January 2021; Published 31 January 2021

Academic Editor: Nicola Baldo

Copyright (c) 2021 Zheng Hu et al. This is an open access article distributed under the Creative Commons Attribution License, which permits unrestricted use, distribution, and reproduction in any medium, provided the original work is properly cited.

\begin{abstract}
Limestone stratum has great anisotropy, which is distributed from large karst caves, pipelines, and faults to small solution pores, and crevices. In this paper, uniaxial compression tests of solution pore and crevice limestones from Mamaya I hydropower station and Ronglai hydropower station are conducted, and the porosity of these limestones is measured. The results show that there is a good power function relationship between compressive strength and the porosity of the solution pore and crevice limestone. Based on the Hoek-Brown criterion, the method for determining mechanical parameters of the solution pore and crevice limestones is proposed, taking the porosity of the rock into consideration. Then, the relationships between the rock mass parameters $m_{b}, s$, and $a$ and the porosity $n$ are deduced. Based on the proposed method, the variation laws of the mechanical parameters of the limestones, including uniaxial compressive strength (UCS), tensile strength, deformation modulus, shear strength parameters are analyzed. The proposed method simplifies the complexity of mechanical parameters selection by quantifying GSI, avoids the subjectivity and uncertainty, and has good reliability and suitability in the pore and crevice limestone stratum, which has a certain guiding significance for the construction of similar sites.
\end{abstract}

\section{Introduction}

Since the rock mass is a complex geological material that undergoes long-term geological processes, the geometrical and mechanical characteristics of the rock mass are fairly complex $[1,2]$. The mechanical parameters of rock the mass, such as the UCS, the deformation modulus, Poisson's ratio, the shear strength parameters, are very crucial parameters that describe the behavior of rock failure and play an increasingly significant role in various rock mechanics engineering [3-6].

In this sense, during the commercial development of capital construction projects, the mechanical properties of rock under loading and unloading conditions are the key to analyze the stability of the slope and have great theoretical significance for the support design of the exacvation slope. There has been substantial effort by engineers to investigate the rock mass mechanical parameters in rock mechanics
[7-11]. Owing to complexities of geological environment and essential properties of joints, accurate measurement of the mechanical parameters is very difficult [12]. While the mechanical properties of rock mass have essentially changed under loading and unloading conditions [13-15], the mechanical properties determined by loading theory are unreliable for unloading slope. However, the mechanical properties of the rock mass with the change of the rock structure in the excavation unloading process are rarely researched at present. In order to obtain the mechanical parameters of the rock mass under loading and unloading conditions, some basic engineering geological conditions (i.e., lithology, porosity) should be considered. The purpose is to better solve strength, deformation, and failure problems of the rock mass in high field stress areas in southwest China. Therefore, it is of great necessity to investigate the mechanical parameters of the rock mass with the change of the 
porosity in the excavation slope, and the results can serve as a better guide to similar excavation projects.

The methods determining the mechanical parameters of the rock mass were put forth by some scholars [16-21], for example, test methods, numerical analysis, displacement back analysis, uncertainty analysis, and empirical analysis. Among them, empirical analysis methods are usually combined with the rock mass classification methods. To quantify the complicated characteristics of the rock mass based on past experience, various rock mass classification methods have been proposed [2]. These classification methods are very important for enhancing the design of the rock mass engineering in practice $[22,23]$. The first work is to identify the score of the quality of the rock mass by setting the quality evaluation index of the rock mass. Then the ranges of the rock mass mechanical parameters are obtained by referring the relevant empirical theories. The evaluation of the quality of the rock mass is essentially regarding the multi-jointed rock with different tendencies as the equivalent continuum, that is, the equivalent mechanical parameters are obtained by determining the quality of the rock mass. However, in practice, the mechanical properties of the rock mass are extremely complex. In view of different rock mass engineering, the rock mass classification method is not always the same. Consequently, rock mass rating (RMR), geological strength index (GSI), rock tunneling quality index, rock mass index, and other empirical methods are widely developed and applied in rock mass engineering [2, 24-26]. The empirical methods are simple to operate, but they have some defects with the characterizing of applicability and limitation. Some expressions of determining the mechanical parameters of rock mass have a certain physical significance, they present contradictory results when they are utilized to assess the mechanical parameters of rock mass with different porosities owing to the shortage of sufficient theoretical backgrounds [27]. Although these rock mass classification methods have been widely used in slope engineering, the degradation effect of the rock structure on the mechanical parameters has not been taken into account.

Except for the discussion mentioned above, the influence of the porosity of the rock on the mechanical properties is rarely considered, especially limestone. The limestone is formed in nature, is a sedimentary rock, and has a bedding structure, which contains not only original fractures but also structural fractures [28]. In particular, the solution pore and crevice limestone is widely distributed in Southwest China, which may have some solution pores and cracks inside rocks. The solution pore and crevices would lead to the deterioration of the limestone. The more dissolution fissures developed in the rock, the worse the slope stability. Therefore, the mechanical parameters of these limestones with solution pore and crevice in practical application are very significant for determining the stability of the slope.

The method should not only possess sufficient physical meaning in evaluating the mechanical parameters of the rock mass but also reflect the whole degradation process of the rock mass with excavation unloading or the internal structure of the rock mass changes on a continuous scale. According to the aforementioned significant issues in this study, the relationship between the porosity of the solution pore and crevice limestone and the UCS are regarded as a reliable and applicable tool for the assessment of the mechanical parameters based on the Hoek-Brown criterion. In this study, uniaxial compression tests of the limestones from two hydropower station and Ronglai hydropower station are conducted. The porosity of these rocks is determined by measuring their specific gravity and dry density. Afterward, the method for determining the mechanical parameters of the rock mass is proposed briefly. Subsequently, considering the porosity of the rock, the variation laws of the mechanical parameters of the limestones are analyzed based on the proposed method. Finally, the outperformance and applicability of the proposed method is validated by comparing with the existing method and test values of internal friction angle for several rock types at two hydropower station.

\section{Test Materials}

2.1. Preparation of Limestone Specimens. The left bank of Mamaya I hydropower station belongs to Guanling County, Guizhou Province, and the right bank belongs to Xingren and Qinglong counties, Guizhou Province, which meanders in the high mountains and valleys in the east of Yunnan Guizhou Plateau. The normal water level of the reservoir, a typical Canyon reservoir, is $585 \mathrm{~m}$, the reservoir capacity is $1.365 \times 10^{8} \mathrm{~m}^{3}$, and the backwater length of the main stream is $45.0 \mathrm{~km}$. The reservoir zone is mainly composed of soluble carbonate rocks, and the geomorphic characteristics are karst low mountain landforms. The exposed strata in the reservoir zone are mainly argillaceous limestone with marl and sandstone of Laishike Formation of Upper Triassic, limestone of Zhuganpo Formation of Middle Triassic, limestone and argillaceous dolomite of Guanling Formation.

The Ronglai hydropower station is located at the junction of Rongdu Town, Ceheng County, and Xinglong Town, Anlong County, Guizhou Province. The overall terrain is a low mountain and hilly area where the watershed of the two rivers gradually decreases from northwest to southwest. The terrain on both sides of the northeast and southwest decreases and enters into the valleys of Nanpan River and Beipan River, respectively. The hydropower station is located in the geomorphic unit of karst trough. The ground elevation of ridges on both sides is $1350-1460 \mathrm{~m}$, and the ground elevation of the trough valley is $1300-910 \mathrm{~m}$. The normal water level of the reservoir is $1180 \mathrm{~m}$, the dam height is $44 \mathrm{~m}$, and the reservoir capacity is about 4.47 million $\mathrm{m}^{3}$. According to the field geological survey, the bedrock in the reservoir zone and both banks is exposed, and the lithology of the exposed stratum is mainly the carbonate rock stratum of Guanling Formation of the Middle Triassic.

In order to reveal the relationship between the strength of the solution pore and crevice limestone and its porosity, we select the medium-thick-bedded limestone of the Middle Triassic Guanling Formation in the Mamaya I hydropower station and the Ronglai hydropower station as the research objects. The thickness of this rock layer is about $235 \sim 515 \mathrm{~m}$. The drilling reveals that the dissolution fractures and holes are relatively developed (as shown in Figure 1). The core of 


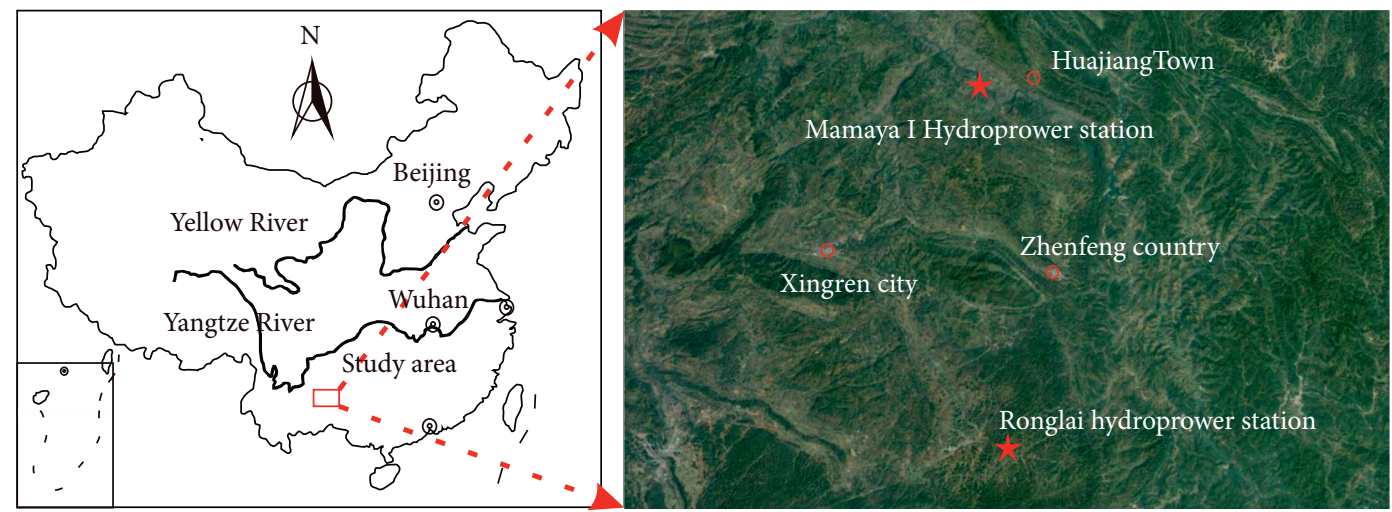

(a)

(b)

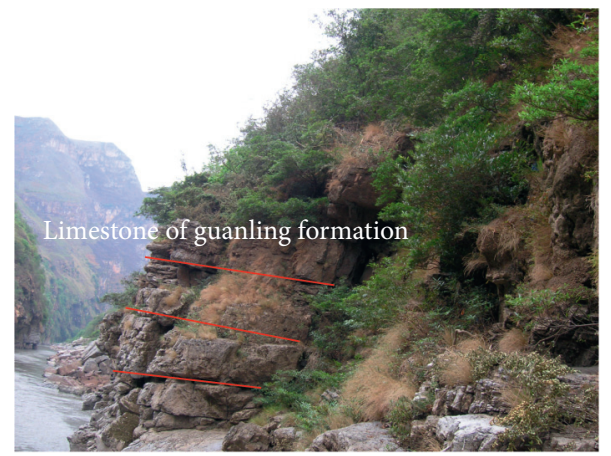

(c)

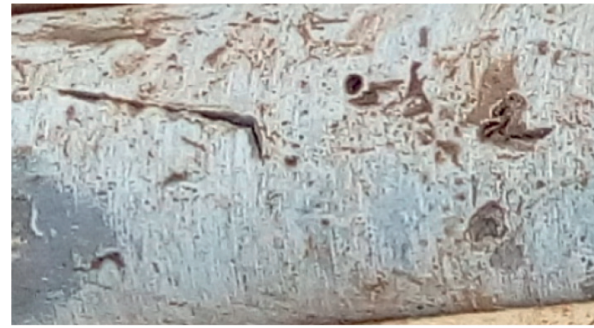

(e)

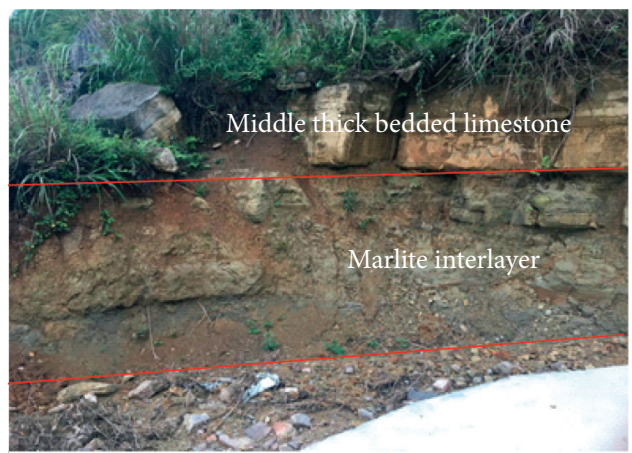

(d)

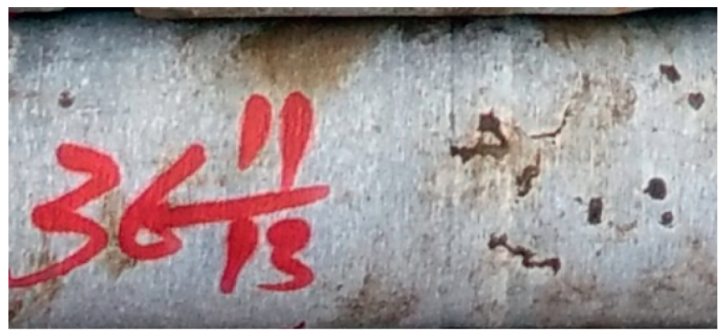

(f)

Figure 1: Sampling site and drilling core of solution pore and crevice limestone: (a) study area; (b) hydropower station location; (c) limestone in Mamaya; (d) limestone in Ronglai; (e) drilling core in Mamaya; (f) drilling core in Ronglai.

the borehole is generally in the form of long column, short column, and a small amount of fragments. The width of the fractures is about $0.2 \sim 1 \mathrm{~cm}$, and the length is $0.3 \sim 3 \mathrm{~m}$. These fractures present as curved, undulating, and rough. There is no calcite or argillaceous filling in these fractures. The diameter of the dissolution holes is $0.2 \sim 2 \mathrm{~cm}$. The distribution of the dissolution fractures and holes is random and uneven. However, the rock mass appears to be in good integrity. These fractures and pores are formed by dissolution during diagenesis. These rock samples are typical dissolution pore and pore limestones. The porosity (expressed as $n$ ) reflects the number of fractures and pores in the rock mass.

2.2. Uniaxial Compressive Test. Uniaxial compression tests of the limestones from Mamaya I hydropower station and Ronglai hydropower station are conducted with a testing machine. During the preparation of rock samples, the geometry of the rock should be ensured to avoid any irregularity that may affect the test results. Coring, as shown in Figures 1(e) and 1(f), is conducted perpendicular to the bedding plane to recover cylindrical rock samples from siltstone blocks. The end of the specimen is polished with a surface grinder to ensure that the ends of the two planes are parallel to each other and perpendicular to the longer dimension. An acoustic wave test is carried out before a uniaxial test to eliminate abnormal rock samples. For uniaxial compression conditions, the confining pressure is set to zero, while the axial stress is increased at a rate of $0.25 \mathrm{kN} / \mathrm{s}$ until the rock specimens fail. In this study, we select 239 groups of solution pore and crevice limestones to conduct uniaxial compression tests.

\subsection{Test Results}

2.3.1. Uniaxial Compression. Uniaxial compression conditions, the physical and mechanical parameters of these limestones from Mamaya I hydropower station are shown in 
TABLE 1: The physical and mechanical parameters of limestones from the Mamaya I hydropower station.

\begin{tabular}{|c|c|c|c|c|c|c|c|c|}
\hline \multirow{2}{*}{ Lithology } & \multirow{2}{*}{ Lists } & \multirow{2}{*}{ Density $\left(\mathrm{kN} / \mathrm{m}^{3}\right)$} & \multicolumn{2}{|c|}{ UCS (MPa) } & \multicolumn{2}{|c|}{ TS $(\mathrm{MPa})$} & \multirow{2}{*}{$E(\mathrm{GPa})$} & \multirow{2}{*}{ Poisson's ratio } \\
\hline & & & Natural & Saturated & Natural & Saturated & & \\
\hline \multirow{2}{*}{$\mathrm{T}_{2} \mathrm{~g}^{2-3-4}$} & Number of samples & 1 & 2 & 2 & 1 & 1 & 1 & 1 \\
\hline & Mean value & 25.28 & 52.3 & 38.3 & 6.85 & 4.13 & 40.2 & 0.29 \\
\hline \multirow{2}{*}{$\mathrm{T}_{2} \mathrm{~g}^{2-3-3}$} & Number of samples & 2 & 5 & 5 & 3 & 3 & 2 & 2 \\
\hline & Mean value & 25.6 & 50.58 & 39.28 & 5.3 & 4.23 & 40.5 & 0.3 \\
\hline \multirow{2}{*}{$\mathrm{T}_{2} \mathrm{~g}^{2-3-2}$} & Number of samples & 3 & 6 & 6 & 2 & 4 & 1 & 1 \\
\hline & Mean value & 26.2 & 40.75 & 33.43 & 5.65 & 2.96 & 38.9 & 0.31 \\
\hline \multirow{2}{*}{$\mathrm{T}_{2} \mathrm{~g}^{2-3-1}(\mathrm{a})$} & Number of samples & 5 & 13 & 12 & 2 & 2 & - & - \\
\hline & Mean value & 25.44 & 48.96 & 30.87 & 6.97 & 5.55 & - & - \\
\hline \multirow{2}{*}{$\mathrm{T}_{2} \mathrm{~g}^{2-3-1}(\mathrm{~b})$} & Number of samples & 13 & 33 & 31 & 13 & 14 & 12 & 13 \\
\hline & Mean value & 26.9 & 89.55 & 64.21 & 7.94 & 5.54 & 55.9 & 0.3 \\
\hline
\end{tabular}

$\mathrm{T}_{2} \mathrm{~g}^{2-3-4}$ represents medium-thick limestone in the fourth sublayer of the third layer of the second member of Guanling formation; $\mathrm{T}_{2} \mathrm{~g}^{2-3-3}$ represents medium-thick spherical micritic limestone in the third sublayer of the third layer of the second member of Guanling formation; $T_{2} \mathrm{~g}^{2-3-2}$ represents mediumthick crystalloid limestone in the second sublayer of the third layer of the second member of Guanling formation; $\mathrm{T}_{2} \mathrm{~g}^{2-3-1}$ (a) represents medium-thick crystalloid limestone in the first sublayer of the third layer of the second member of Guanling formation; $\mathrm{T}_{2} \mathrm{~g}^{2-3-1}(\mathrm{~b})$ represents medium-thick limestone in the first sublayer of the third layer of the second member of Guanling formation.

Table 1. Table 1 shows the density, the UCS, tensile strength (TS), elastic modulus (E), and Poisson's ratio of five types of rocks. For these rock samples, mean value of their density is $25.28-26.9 \mathrm{kN} / \mathrm{m}^{3}$. A total of 115 sets of uniaxial compression tests for five types of rocks are obtained, including 59 sets of data for natural conditions and 56 sets of data for saturated conditions. For the same rock type, the natural UCS is obviously higher than the saturated UCS. The mean value of the natural UCS is $40.75-89.55 \mathrm{MPa}$, while the mean value of the saturated UCS is $30.87-64.21 \mathrm{MPa}$. Overall, the ratio of the saturated UCS to the natural UCS is $63.05 \%-82.04 \%$. A total of 45 sets of tensile strength tests for four types of rocks are conducted, including 21 sets of data for natural conditions and 24 sets of data for saturated conditions. For the same rock type, the natural TS is also obviously higher than the saturated TS. The mean value of the natural TS is $5.3-7.94 \mathrm{MPa}$, while the mean value of the saturated TS is $2.96-5.55 \mathrm{MPa}$. Overall, the ratio of the saturated TS to the natural TS is $52.39 \%-79.81 \%$. In addition, the ratio of the natural UCS to the natural TS is $0.09-0.14$, and the ratio of the saturated UCS to the saturated TS is $0.09-0.18$. These variations obey the general laws [29], which validate that the test results are effective. For deformation parameters, the mean value of the elastic modulus is $38.9-55.9 \mathrm{GPa}$, while the mean value of the Poisson's ratio is $0.29-0.31$.

Uniaxial compression conditions, the physical and mechanical parameters of these limestones from Ronglai hydropower station, are shown in Table 2. Table 2 shows the density, water absorption, the UCS, the elastic modulus, and the Poisson's ratio of three types of rocks. For these rock samples, the mean value of their density is $25.28-26.9 \mathrm{kN} / \mathrm{m}^{3}$ and the mean value of the water absorption is $0.29-0.37$. A total of 124 sets of uniaxial compression test for three types of rocks are obtained. For the same rock type, the natural UCS is obviously higher than the saturated UCS. The mean value of the natural UCS is $41-74.1 \mathrm{MPa}$, while the mean value of the saturated UCS is $25.3-43.5 \mathrm{MPa}$. Overall, the ratio of the saturated UCS to the natural UCS is $58.70 \%-66.72 \%$. For the deformation parameters, the mean value of the elastic modulus is 46.4-64.1 GPa, while the mean value of the Poisson's ratio is $0.24-0.26$. Noteworthy, due to the limited number of samples, the saturated UCS in $\mathrm{T}_{2} \mathrm{~g}^{1-2}$ is obviously lower. According to relevant experience, the lithology of the rock sample is medium-hard rock, so the saturated UCS should be between $30 \sim 60 \mathrm{MPa}$, while the test result is not up to $30 \mathrm{MPa}$, which is mainly because the rock sample of this group is close to the surface, the weathering degree is high, and the fractures are relatively developed. Therefore, the test results should be used as a reference index, and the relatively reasonable and reliable physical and mechanical parameters of the rocks should be determined based on the comprehensive analogy of related projects.

2.3.2. Relationship between the UCS and Porosity. As mentioned above, the physical and mechanical parameters of the solution pore and crevice limestones are obtained. According to these results, the porosity of the rock samples can be determined based on the specific gravity and the dry density of these rock samples. Herein, the specific gravity of the rock refers to the dry specific gravity of rock divided by the product of the volume of the rock (excluding pores) and the bulk density of water at $4^{\circ} \mathrm{C}$, expressed as

$$
G_{s}=\frac{W_{s}}{V_{s} \gamma_{w}},
$$

where $G_{s}$ is the specific gravity, $W_{s}$ is the gravity of the rock in absolute dry condition, $V_{s}$ is the volume of the rock (excluding pores), and $\gamma_{W}$ is the bulk density of water at $4^{\circ} \mathrm{C}$, $\gamma_{W}=10 \mathrm{kN} / \mathrm{m}^{3}$. 
TABLE 2: The physical and mechanical parameters of these limestones from Ronglai hydropower station.

\begin{tabular}{llcccccc}
\hline Lithology & Lists & Density $\left(\mathrm{g} / \mathrm{cm}^{3}\right)$ & Water absorption (\%) & Natural UCS (MPa) & Saturated UCS (MPa) & $E(\mathrm{GPa})$ & Poisson's ratio \\
\hline \multirow{2}{*}{$\mathrm{T}_{2} \mathrm{~g}^{1-3-1}$} & Range value & 2.83 & 0.37 & $73.2 \sim 75.1$ & $42.3 \sim 44.7$ & 63.3 & 0.24 \\
& Mean value & 2.83 & 0.37 & 74.1 & 43.5 & 63.3 & 0.24 \\
\hline \multirow{2}{*}{$\mathrm{T}_{2} \mathrm{~g}^{1-2}(\mathrm{a})$} & Range value & 2.82 & 0.29 & $57.1 \sim 61.4$ & $35.0 \sim 44.0$ & 64.1 & 0.25 \\
& Mean value & 2.82 & 0.29 & 59.2 & 39.5 & 64.1 & 0.25 \\
\hline \multirow{2}{*}{$\mathrm{T}_{2} \mathrm{~g}^{1-2}$ (b) } & Range value & 2.81 & 0.30 & $34.5 \sim 47.5$ & $23.8 \sim 26.9$ & 46.4 \\
& Mean value & 2.81 & 0.30 & 41.0 & 25.3 & 0.26 \\
\hline
\end{tabular}

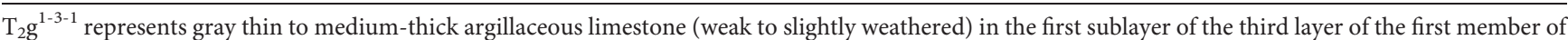
Guanling formation; $\mathrm{T}_{2} \mathrm{~g}^{1-2}$ (a) represents gray thin to medium-thick argillaceous limestone (slightly weathered) in the second layer of the first member of Guanling formation; $\mathrm{T}_{2} \mathrm{~g}^{1-2}$ (b) represents gray thin to medium-thick argillaceous limestone (weak weathered) in the second layer of the first member of Guanling formation.

The porosity of the rock is defined as the ratio of the volume of pores and the total volume of the rock (including pores). The porosity of the rock is calculated by the specific gravity and dry density, expressed as

$$
n=\frac{100\left(G_{s}-\rho_{d}\right)}{G_{s}}
$$

where $n$ is the porosity of the rock and $\rho_{d}$ is the dry density of the rock.

To summarize, the porosity for 247 sets of limestones is obtained by equation (2), and its range is $0-15 \%$. In this study, the range of the porosity is divided into three parts, including $0-0.5 \%, 0.5 \%-5 \%$, and $5 \%-15 \%$, to discuss the relationships between the UCS and the porosity of the rocks.

There are 14 sets of limestones under natural conditions and 21 sets of limestones under saturated conditions when the range of the porosity of these rocks is $0-0.5 \%$. Figure 2 presents the variation of the UCS with the porosity under natural and saturated conditions. Both the natural UCS and the saturated UCS change little with the porosity, which implies that when the porosity of the rocks is less than $0.5 \%$, the rocks are basically intact, and the strength of the rocks is only affected by their own mineral composition and structure. For these sets of the limestones, the mean value of the natural UCS is $84.6 \mathrm{MPa}$ and the mean value of the saturated UCS is $75.8 \mathrm{MPa}$.

There are 87 sets of limestones under natural conditions and 81 sets of limestones under saturated conditions when the range of the porosity of these rocks is $0.5-5 \%$. Figure 3 presents the variation of the UCS with the porosity under natural conditions, and Figure 4 presents the variation of the UCS with the porosity under saturated conditions. Obviously, both the natural UCS and the saturated UCS decrease sharply with the porosity, and the decreasing rate is becoming smaller and smaller with the increase in the porosity. Especially, the decreasing rate of the rocks is the largest when $n=0.5 \% \sim 1 \%$, which indicates that the strength of the rock will deteriorate obviously when the solution pore and crevice of limestone develops to a certain extent. The main reason is that with the initial increase of the porosity of the limestone, the cohesion between grains decreases, and the UCS decreases under the binding action of frozen pore water and capillary water. As the porosity of the limestone decreases slowly to a certain extent, the binding force of pore water and capillary water in limestone does not increase significantly, and the increase of the cohesion between grains is limited. Therefore, the growth rate of the UCS becomes slow.

It can be seen from Figures 3 and 4 that the relationships between the UCS and the porosity under natural and saturated conditions have a good power function characteristic. Therefore, in this study, the power function $\left(y=a x^{b}\right)$ is selected to fit their relationships. Apparently, the fitting correlations between them under these conditions are very good. Furthermore, the fitting parameter $b$ is approximately close to -0.5 . According to the characteristics of the power function, when $b=-0.5$ and $x=1 \%$, the fitting parameter $a$ can be calculated. Thus, the physical meaning of the fitting parameter $a$ is regarded as the UCS when the porosity of the rock is $1 \%$.

Generally, the compressive strength of the rock obtained in the laboratory cannot represent the compressive strength of the rock mass on a macroscopic scale. For most limestones in Southwest China, the anisotropy of the rocks is mainly manifested in the large karst caves, sinkholes, and underground river pipelines. Actually, the limestone belongs to hard rock with solid stratigraphic framework. Generally, limestone stratum has good engineering characteristics without large karst caves, sinkholes, underground river pipelines, etc. Therefore, in the limestone stratum without large karsts, sinkholes, and underground river pipelines, it is assumed that the mathematical relationship between the strength of the rock and its porosity can be extended to the rock mass in a macroscopic scale. Their mathematical relationship can be established when the range of the porosity of the rock is $0.5 \% \sim 5 \%$.

$$
R_{\mathrm{cm}}=R_{1} n^{-0.5}(0.5 \% \leq n \leq 5 \%)
$$

where $R_{\mathrm{cm}}$ is the UCS of the solution pore and crevice limestones; $R_{1}$ is the UCS of the rock when $n=1 \%$. 


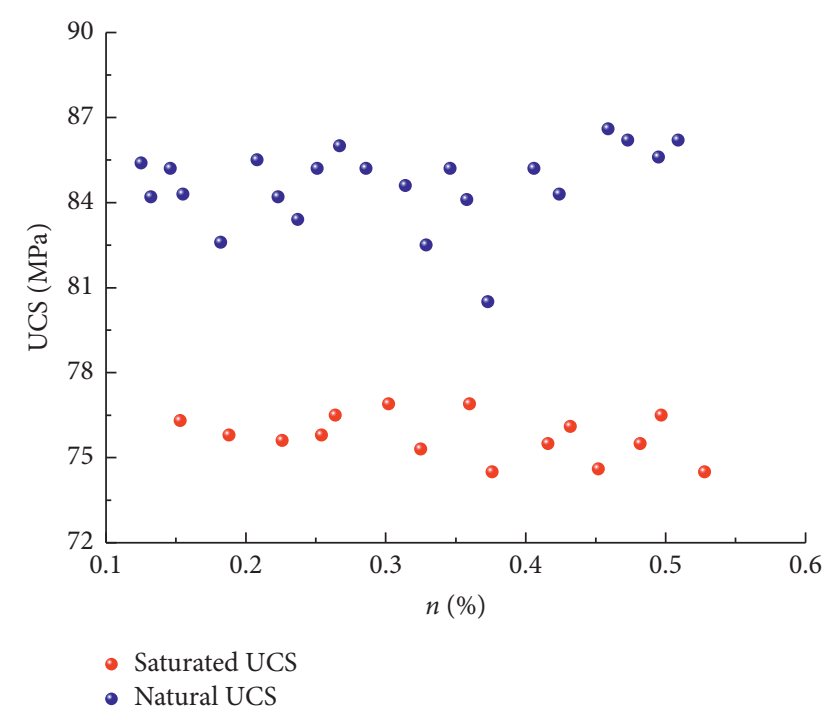

FIGURE 2: The relationship between the UCS and the porosity $(n=0 \sim 0.5 \%)$.

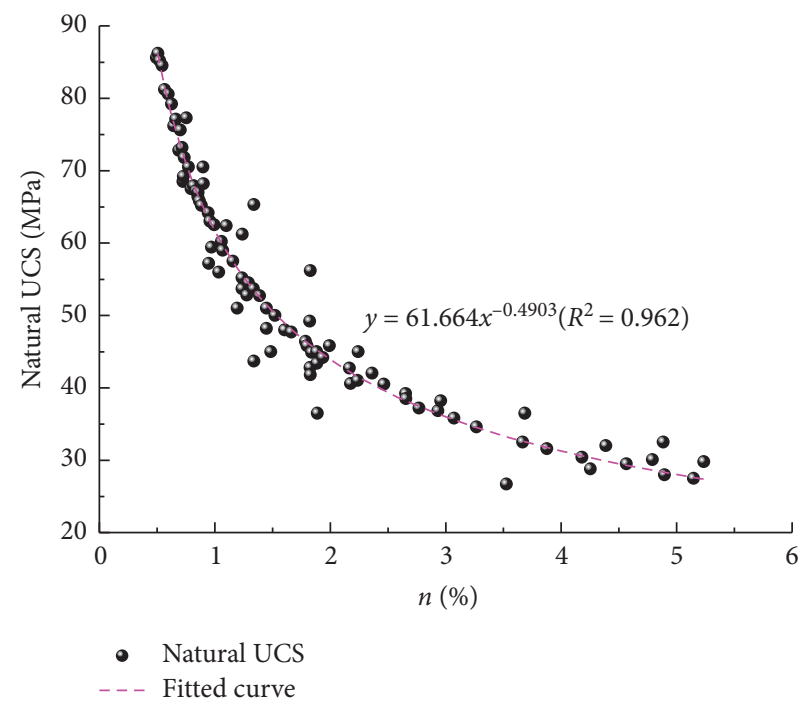

FIGURE 3: The fitted curves of the natural UCS and the porosity $(n=0.5 \% \sim 5 \%)$.

Substituting equation (2) into equation (3), the UCS of the solution pore and crevice limestones can be obtained, as follows:

$$
R_{\mathrm{cm}}=R_{1}\left(\frac{100\left(G_{s}-\rho_{d}\right)}{G_{s}}\right)^{(1 / 2)}
$$

There are 18 sets of limestones under natural conditions and 18 sets of limestones under saturated conditions when the range of the porosity of these rocks is $5-15 \%$. Figure 5 presents the variation of the UCS with the porosity under natural and saturated conditions when $n=5 \% \sim 15 \%$. Both the natural UCS and the saturated UCS decrease slowly with the porosity, indicating that when the porosity of the rocks is

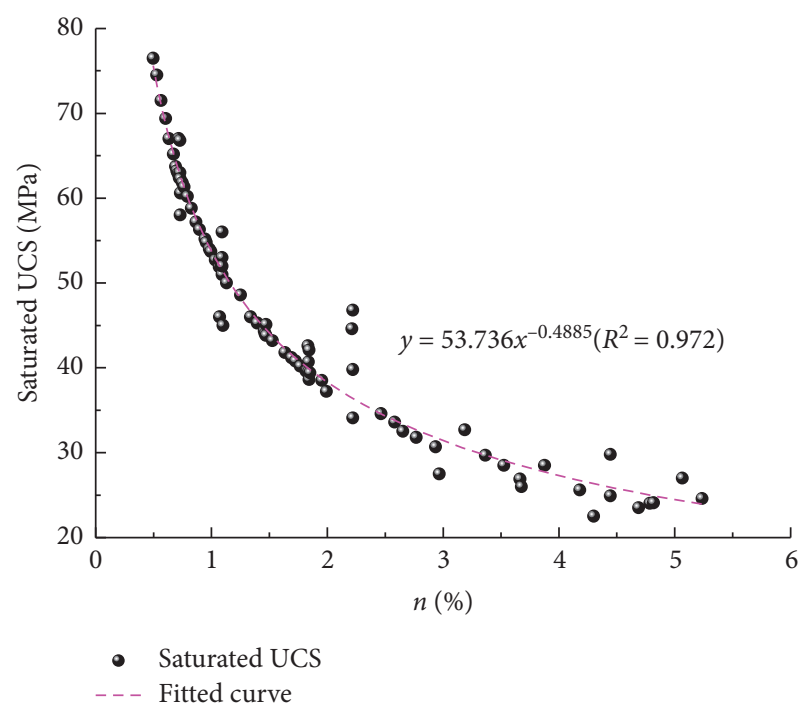

FIgURE 4: The fitted curves of the saturated UCS and the porosity $(n=0.5 \% \sim 5 \%)$.

more than 5\%, the UCS of the rocks tends to residual strength, and the strength of the rocks is mainly controlled by the characteristics of pores and fissures and their occlusal effects. For these sets of limestones, the mean value of the natural UCS is $27.7 \mathrm{MPa}$ and the mean value of the saturated UCS is $25.2 \mathrm{MPa}$.

\section{Methods}

3.1. Proposed Methods. Many improvements have been made to Hoek-Brown criterion since it was first put forth [30]. When estimation approach of $m$ and $s$ values were developed from the RMR by Bieniawski to the GSI by Hoek and Brown [31], the application range of the Hoek-Brown criterion was accordingly enlarged from hard rock mass to the rock mass of poor quality. The Hoek-Brown criterion is formulized as [29, 32, 33]:

$$
\sigma_{1}=\sigma_{3}+\sigma_{c}\left(m_{b} \frac{\sigma_{3}}{\sigma_{c}}+s\right)^{a},
$$

where $\sigma_{c}$ is the UCS; $\sigma_{1}$ is the maximum principal stress; $\sigma_{3}$ is the minimum principal stress; $m_{b}, s$, and $a$ are empirical parameters for the rock mass; $m_{b}$ reflects soft or hard degree of the rock mass with the value ranging from 0.0000001 to 25 , respectively, corresponding to seriously disturbed rock mass and undisturbed rock mass; $s$ reflects the degree of the rock mass fragmentation, the lower limit 0 and upper limit 1 of which make the same sense with those of $m_{b}$.

The study in [9] gave the GSI, mainly to describe the mechanical properties of rock mass. The rock mass scaling relationships for $m_{b}, s$, and $a$ were reported by depending on the value of the GSI, as defined by the equation:

When the GSI $>25$, the following equations can be established: 


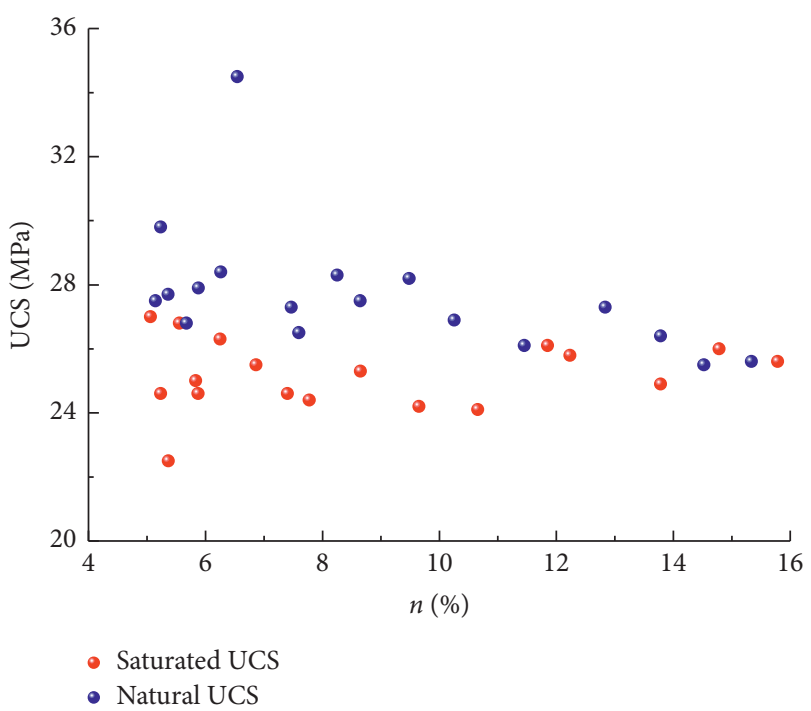

FIGURE 5: The relationship between the UCS and the porosity $(n=5 \% \sim 15 \%)$.

$$
\left\{\begin{aligned}
m_{b} & =\exp \left(\frac{\mathrm{GSI}-100}{28}\right) m_{i}, \\
s & =\exp \left(\frac{\mathrm{GSI}-100}{9}\right), \\
a & =0.5,
\end{aligned}\right.
$$

where $m_{i}$ is a constant of intact rock in the Hoek-Brown criterion, gained in the similar way with the UCS by experimental means.

When the GSI $<25$, the following equations can be established:

$$
\left\{\begin{aligned}
m_{b} & =\exp \left(\frac{\mathrm{GSI}-100}{28}\right) m_{i}, \\
s & =0 \\
a & =0.65-\frac{\mathrm{GSI}}{200}
\end{aligned}\right.
$$

According to equation (5), if the confining pressure is zero, the UCS can be determined, as follows:

$$
\sigma_{\mathrm{cm}}=\sigma_{c} s^{a}
$$

Then, substituting equation (3) into equation (8), and improved equation ( 8$)$ is formed:

$$
R_{1} n^{(-0.5)}=\sigma_{c} s^{a}
$$

According to equations (5) and (6), the range of the parameter $a$ is $0.5-0.65$. In this study, for the solution pore and crevice limestone, the parameter $a$ is set to 0.5 . Therefore, the parameter $s$ can be calculated based on equation (9), as follows:

$$
s=\left(\frac{R_{1}}{\sigma_{c}}\right)^{2} \frac{1}{n}
$$

Then substituting equation (10) into equation (6), the GSI can be obtained in another form, as follows:

$$
\mathrm{GSI}=9 \ln \left[\left(\frac{R_{1}}{\sigma_{c}}\right)^{2} \frac{1}{n}\right]+100
$$

Yet, abstract is difficult to quantify, and the GSI is difficult to work in practical engineering. In order to resolve the embarrassment, the porosity of the rock is introduced followed by substituting GSI; then the parameters $m_{b}, s$, and $a$ are, respectively, expressed as follows:

$$
\left\{\begin{aligned}
m_{b} & =\left[\left(\frac{R_{1}}{\sigma_{c}}\right)^{2} \frac{1}{n}\right]^{(9 / 28)} m_{i}, \\
s & =\left(\frac{R_{1}}{\sigma_{c}}\right)^{2} \frac{1}{n} \\
a & =0.5 .
\end{aligned}\right.
$$

According to equation (12), the variation laws of the parameters $m_{b}$ and $s$ with the porosity of the rock are determined. By combining field investigation and test data, some basic parameters of the solution pore and crevice limestones in the Mamaya I hydropower station and Ronglai hydropower station are acquired. In this study, $\sigma_{c}=75 \mathrm{MPa}, R_{1}=53.736 \mathrm{MPa}, m_{i}=10$. In addition, the range of the porosity of the rock is limited to $0.5 \%-15 \%$. Figure 6 shows the change curve of the parameters $m_{b}$ and $s$ with the porosity of the rock. It indicates that both the parameters $m_{b}$ and $s$ nonlinearly decrease with the porosity, and reduce ranges of them from low porosity to high porosity are $66.7 \%$ and $96.7 \%$, respectively. Especially, the decreasing rates of the parameters $m_{b}$ and $s$ with the porosity of the rock are the largest when $n=0.5 \% \sim 1 \%$, then they gradually decrease when $n=1 \% \sim 5 \%$. Finally, their decreasing rates gradually slow down, even tend to be constant when $n=5 \% \sim 15 \%$.

\subsection{Influence of the Porosity on the Mechanical Parameters}

3.2.1. Degradation of Compressive Strength and Tensile Strength. It is assumed that $\sigma 3=0$ is made for solving the $\sigma_{\mathrm{cm}}$ in equation (5), thus the expression of the UCS is obtained in equation (8). Similarly, to determine the TS by Hoek-Brown criterion, the TS of the rock is formulized based on trial and error [34], as follows:

$$
R_{\mathrm{tm}}=\frac{1}{2} \sigma_{c}\left(m_{b}-\sqrt{m_{b}^{2}+4 s}\right) .
$$

Then substituting equation (12) into equation (13), the TS can be obtained in another form, as follows: 


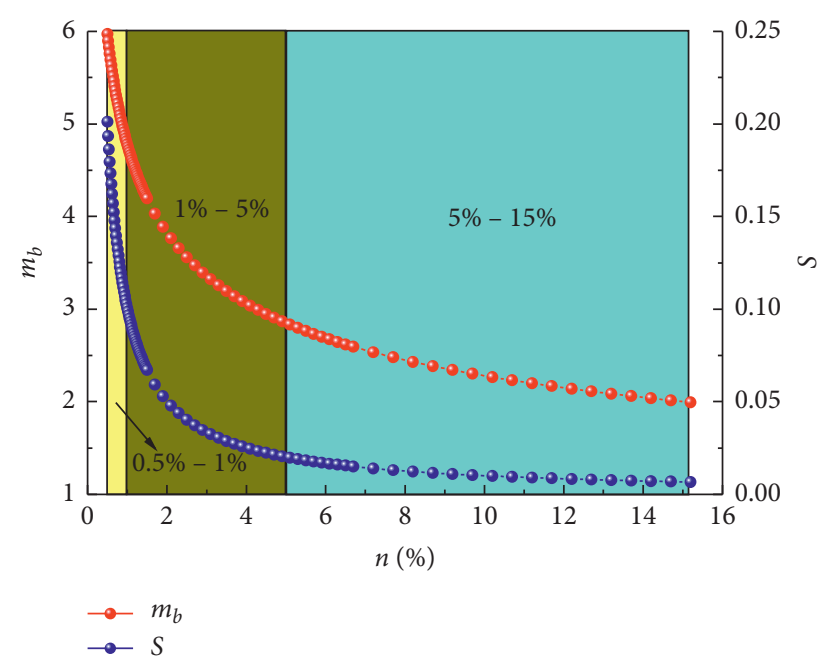

Figure 6: The change curve of $m_{b}$ and $s$ with porosity.

$$
\begin{aligned}
R_{\mathrm{tm}} & =\frac{1}{2} \sigma_{c}\left[\left[\left(\frac{R_{1}}{\sigma_{c}}\right)^{2} \frac{1}{n}\right]^{(9 / 28)} m_{i}\right. \\
& -\sqrt{\left.\left[\left[\left(\frac{R_{1}}{\sigma_{c}}\right)^{2} \frac{1}{n}\right]^{(9 / 28)} m_{i}\right]^{2}+4\left(\frac{R_{1}}{\sigma_{c}}\right)^{2} \frac{1}{n}\right]} .
\end{aligned}
$$

According to equations (8) and (14), the changes in the UCS and the TS are obtained by the proposed method, shown in Figure 7. It records the variation of both present descending trends generally, and from low porosity to high porosity, the UCS and the TS decline to $81.87 \%$ and $90 \%$, respectively. Similarly, the decreasing rates of the UCS and the TS with the porosity of the rock are the largest when $n=0.5 \% \sim 1 \%$, then gradually decrease when $n=1 \% \sim 5 \%$. Finally, their decreasing rates gradually slow down, even tend to be constant when $n=5 \% \sim 15 \%$. More concretely, in the region of $0.5 \% \sim 1 \%$, the UCS and the TS decline to $29.29 \%$ and $37.45 \%$, respectively. In the region of $1 \% \sim 5 \%$, the UCS and the TS decline to $55.34 \%$ and $66.24 \%$, respectively. In the region of $5 \% \sim 15 \%$, the UCS and the TS decline to $41.67 \%$ and $53.02 \%$, respectively. Noteworthy is that the greater the negative value, the greater the tensile strength. Therefore, the rock mass properties evidently degrade with an increase in the porosity of the rock.

3.2.2. Degradation of Deformation Modulus. As with the procedure [34], excavation degradation factor (represented as $D_{e}$ in formulas) is introduced, which yields the deformation modulus as follows [31, 35]:

When $\sigma_{c}>100 \mathrm{MPa}$, the following equation can be established:

$$
E_{m}=\left(1-\frac{D_{e}}{2}\right) \sqrt{\frac{\sigma_{c}}{100}} 10^{((\mathrm{GSI}-10) / 40)}
$$

When $\sigma_{c} \leq 100 \mathrm{MPa}$, the following equation can be established:

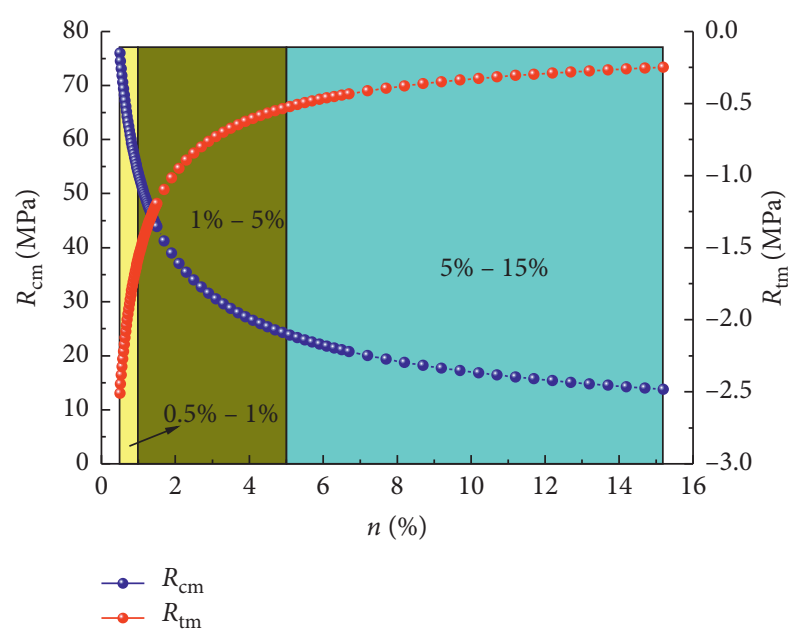

FIgURE 7: Both $R_{\mathrm{cm}}$ and $R_{\mathrm{tm}}$ vary with the porosity.

$$
E_{m}=\left(1-\frac{D_{e}}{2}\right) 10^{((\mathrm{GSI}-10) / 40)}
$$

Since the UCS of the solution pore and crevice limestones is less than $100 \mathrm{MPa}$ in the Mamaya I hydropower station and Ronglai hydropower station, equation (16) is utilized to determine the deformation modulus. Furthermore, $D_{e}$ is assumed to be 0 due to the good integrity of the limestones. Subsequently, substituting the porosity for GSI in equation (16), the deformation modulus is formulized as

$$
E_{m}=\sqrt{\frac{\sigma_{c}}{100}} 10\left(\left(9 \ln \left[\left(R_{1} / \sigma_{c}\right)^{2}(1 / n)\right]+90\right) / 40\right) .
$$

Figure 8 depicts the variation of the deformation modulus obtained by the proposed method, in which a nonlinear negative correlation is witnessed, drop from 156.12 GPa to $26.62 \mathrm{GPa}$. That is, the deformation modulus reduces to $82.95 \%$ from low porosity to high porosity, indicating its degradation. Similarly, the decreasing rate of the deformation modulus with the porosity of the rock is the largest when $n=0.5 \% \sim 1 \%$, then it gradually decreases when $n=1 \% \sim 5 \%$. Finally, its decreasing rate gradually slows down when $n=5 \% \sim 15 \%$. More concretely, in the region of $0.5 \% \sim 1 \%$, the deformation modulus declines to $30.13 \%$. In the region of $1 \% \sim 5 \%$, the deformation modulus declines to $56.88 \%$. In the region of $5 \% \sim 15 \%$, the deformation modulus declines to $43.4 \%$. The result agrees well with in-situ measurement of the deformation modulus during the process of the porosity increasing.

3.2.3. Degradation of Shear Strength Parameters. In order to get the shear strength parameters (e.g., cohesion $c$, internal friction angel $\varphi$ ), both the $\sigma 1$ and $\sigma 3$ are linearly fitted into formula $\sigma 1=k \sigma 3+b$ under peak strength and residual strength, respectively, based on the combination of Mohr-Coulomb criteria and the regression method. Then the expressions read as 


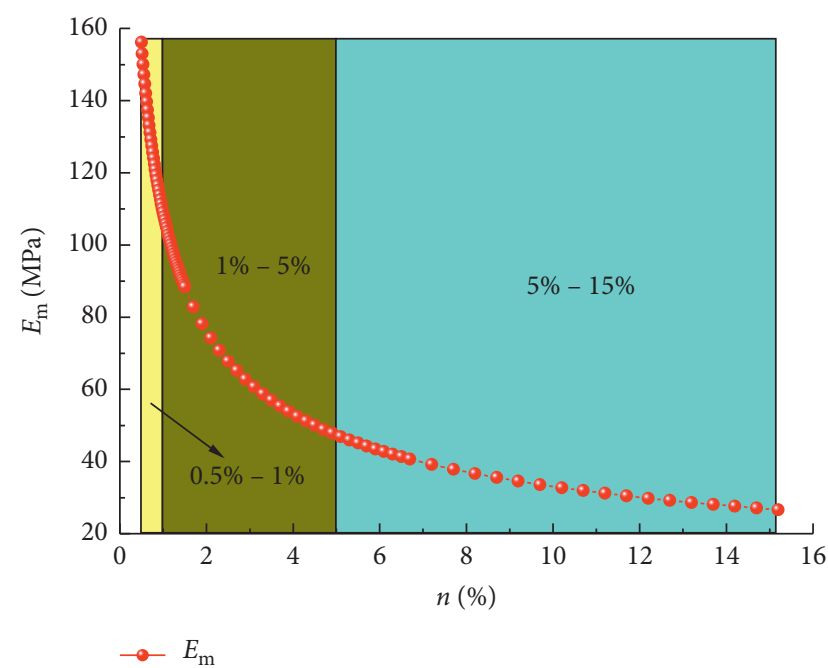

FIGURE 8: $E_{m}$ varies with the porosity.

$$
\begin{gathered}
\varphi=\arcsin \left(\frac{k-1}{k+1}\right), \\
c=\frac{b(1-\sin \varphi)}{2 \cos \varphi} .
\end{gathered}
$$

Depending on the HB strength criteria, the envelope slope of which has been quantified by taking the derivative of equation (5) with respect of $\sigma 3$ :

$$
k=\frac{d \sigma_{1}}{d \sigma_{3}}=1+a m_{b}\left(m_{b} \frac{\sigma_{3}}{\sigma_{c i}}+s\right)^{a-1} .
$$

Substituting $k$ into equation (20), we obtain the shear strength parameters of the rock mass:

$$
\left\{\begin{array}{l}
\varphi_{m}=\arcsin \frac{a m_{b}\left(m_{b}\left(\sigma_{3} / \sigma_{c i}\right)+s\right)^{a-1}}{a m_{b}\left(m_{b}\left(\sigma_{3} / \sigma_{c i}\right)+s\right)^{a-1}+2}, \\
c_{m}=\frac{\sigma_{c m}\left(1-\sin \varphi_{m}\right)}{2 \cos \varphi_{m}},
\end{array}\right.
$$

where $m_{b}, s$, and $a$ are calculated based on equation (12).

There are two cases of degradation laws of the shear strength parameters based on equation (21). One is the variation of the shear strength parameters of the rock mass with the confining pressure $\sigma 3$ in the process of the porosity increasing. The other is the variation of the shear strength parameters of the rock mass with the porosity in the process of the confining pressure increasing. In this study, we select the porosity as an independent variable, and the shear strength parameters as the dependent variable. When drawing $\varphi_{m}-n$ and $c_{m}-n$ curves, $\sigma 3$ is regarded as the values $-2 \mathrm{MPa},-1 \mathrm{MPa}, 0 \mathrm{MPa}, 1 \mathrm{MPa}$, $2 \mathrm{MPa}, 5 \mathrm{MPa}, 10 \mathrm{MPa}, 30 \mathrm{MPa}$ to comparatively discuss. Noteworthy, the rock is in the tensile state when the confining pressure is $-2 \mathrm{MPa}$ or $-1 \mathrm{MPa}$.

Clearly as seen in Figures 9 and 10, under compression conditions, the shear strength parameters suffer different levels of degeneration as the porosity increases, but the rangeability of $\varphi_{m}$ is less than $c_{m}$. This is because the increase

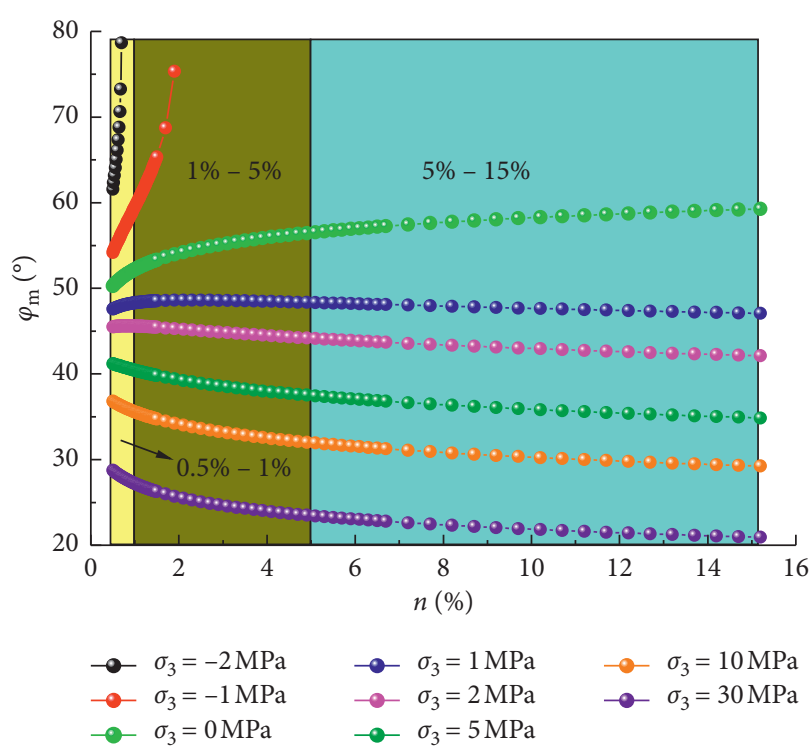

Figure 9: The change laws of $\varphi_{m}$ with the porosity and $\sigma_{3}$.

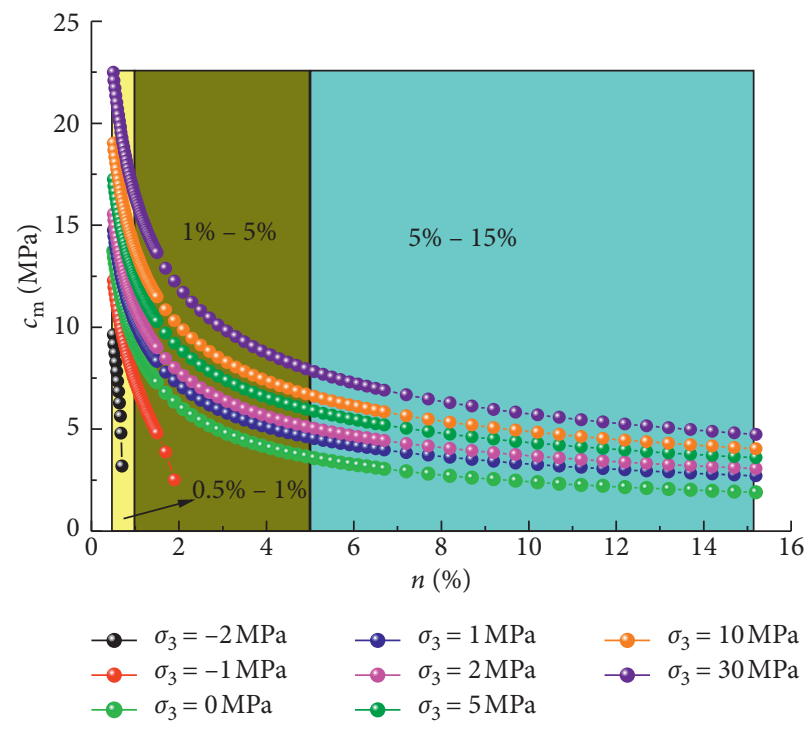

Figure 10: The change laws of $c_{m}$ with the porosity and $\sigma_{3}$.

of the porosity in the rock mass intensifies the development and expansion of internal microcracks, which leads to the weakening or disappearance of intergranular force and the decrease of $\mathrm{cm}$ value. The value as well as rangeability of $\varphi_{m}$ is smaller with the increase of the confining pressure in the same value of $n$; however, the larger the value of $c_{m}$, the smaller the rangeability. The difference of $\varphi_{m}$ between two high confining pressures is very small and their $\varphi_{m}$ has the same variation tendency, and so does $\mathrm{cm}$. Moreover, $\varphi_{m}$ owns a larger change sensitivity than $c_{m}$ in low stress areas, indicating that the internal friction angle is more susceptible, by comparison, to the porosity of the rocks.

Under tensile conditions, $\varphi_{m}$ increases rapidly with the porosity, then the rock fails rapidly. The porosity of the rock is controlled by the TS. On the one hand, the tension action of the rock mass intensifies the development and expansion of internal 
microcracks, which increases the porosity of the rock mass. On the other hand, the tension of microcracks increases the roughness of the structural plane of the rock mass, which shows an increase in internal friction force. However, the "enhancement" of shear strength properties of the rock mass caused by the increase of internal friction force is far less than that of "weakening" of the rock mass properties caused by the tension of the fractures. Furthermore, the rock is very sensitive to this tension action, so the rock shows rapid fracture instability.

Under tensile conditions, $\mathrm{cm}$ decreases rapidly with the porosity, then the rock fails rapidly. The porosity of the rock is controlled by the TS during failure. The tension action of the rock mass intensifies the development and expansion of internal microcracks, which leads to the weakening or disappearance of intergranular force and the decrease of cohesion.

\section{Application}

According to the proposed method in this study, the mechanical parameters of each group of rocks in different boreholes can be obtained, substituting equation (12) into equations (17) and (21). In order to validate the rationality of the method for determining the mechanical parameters of the rock mass, the rock mass in the Mamaya I hydropower station and Ronglai hydropower station mentioned above is still used as the case study. Furthermore, the method to determine the mechanical parameters based on the ultrasonic velocity is compared. The comparison method is illustrated as follows.

The empirical parameters in the Hoek-Brown criterion are obtained based on the GSI. If the GSI and the UCS are known, the shear strength parameters can be determined. The determination of the GSI has three methods [36]: the first is to directly observe and measure the outcrop of the rock mass in the field, and compare it with GSI chart; the second is to estimate by the value of the RMR; and the third method to obtain the GSI is to estimate the volume of the rock mass and joint plane conditions. Therefore, it can be seen that the GSI can be easily obtained in the current hydropower projects, from the perspective of the reliability of the application.

Xia et al. [37] proposed the estimation method of the rock mass mechanical parameters based on the ultrasonic velocity of the rock mass. Firstly, the relationship between the ultrasonic velocity and the RMR was provided, as expressed:

$$
\mathrm{RMR}_{89}=15 V_{p}-2.5,
$$

where $\mathrm{RMR}_{89}$ is the rock mass rating proposed by $\mathrm{Z}$. T. Bieniawski in 1989; $V_{p}$ is the ultrasonic velocity.

Combined with the $\mathrm{RMR}_{89}$ and the GSI, the following quantitative relationship can be acquired when the $\mathrm{RMR}_{89}>23$ [38]:

$$
\mathrm{GSI}=\mathrm{RMR}_{89}-5
$$

Substituting equation (22) into equation (23), the following equation can be obtained:

$$
\mathrm{GSI}=15 V_{p}-7.5 \text {. }
$$

Substituting equation (24) into equations (5)-(7), (13), and (21), the mechanical parameters of the rock mass, including the deformation modulus, the internal friction angle, and the cohesion, can be determined based on the ultrasonic velocity method.

Since the GSI is related to the rock mass structure, the interlocking state of rock blocks in the rock mass and the characteristics of the structural plane are not considered, the influence of excavation and blasting on the GSI is also not considered. Therefore, the $V_{p}$ in equation (24) refers to the ultrasonic velocity of the undisturbed rock mass, which is only suitable for the rock mass with an ultrasonic velocity greater than $1700 \mathrm{~m} / \mathrm{s}$, but it is not suitable for the rock mass with poor quality.

In view of the above two cases, the data of acoustic tests of some boreholes in the Mamaya I hydropower station and Ronglai hydropower station are collected, as shown in Tables 3 and 4. Table 3 presents the elevation of the acoustic tests and the ultrasonic velocity of some of the boreholes in the Mamaya I hydropower station. According to the statistics of the acoustic test of 14 boreholes in the dam site area, it is mainly $\mathrm{T}_{2} \mathrm{~g}^{2-3-2}, \mathrm{~T}_{2} \mathrm{~g}^{2-3-1}$, and $\mathrm{T}_{2} \mathrm{~g}^{2-2}$ strata. The test results indicate that the ultrasonic velocity of weakly weathered rock mass $\left(\mathrm{T}_{2} \mathrm{~g}^{2-3-2}\right.$ ) ranges from $2500 \mathrm{~m} / \mathrm{s}$ to $5570 \mathrm{~m} / \mathrm{s}$, with an average of $4380 \mathrm{~m} / \mathrm{s}$. The rock mass with low $V_{p}$ is mostly distributed in the fracture development zone or dissolution fracture zone. The ultrasonic velocity of slightly fresh rock mass $\left(\mathrm{T}_{2} \mathrm{~g}^{2-3-2}\right)$ ranges from $3520 \mathrm{~m} / \mathrm{s}$ to $6500 \mathrm{~m} / \mathrm{s}$, with an average of $5843 \mathrm{~m} / \mathrm{s}$. The integrity of weakly rock mass is poor, and that of slightly fresh is good. The ultrasonic velocity of weakly weathered rock mass $\left(\mathrm{T}_{2} \mathrm{~g}^{2-}\right.$ ${ }^{3-1}$ ) ranges from $1418 \mathrm{~m} / \mathrm{s}$ to $6260 \mathrm{~m} / \mathrm{s}$, with an average of $5016 \mathrm{~m} / \mathrm{s}$. The rock mass with low $V_{p}$ is mostly distributed in the fracture development zone or the dissolution fracture zone. The ultrasonic velocity of slightly fresh rock mass $\left(\mathrm{T}_{2} \mathrm{~g}^{2-3-1}\right)$ ranges from $1980 \mathrm{~m} / \mathrm{s}$ to $6600 \mathrm{~m} / \mathrm{s}$, with an average of $5117 \mathrm{~m} / \mathrm{s}$. The rock mass with low $V_{p}$ is mostly a thin-layer intercalated with thin-layer limestone or crystal cave limestone. The integrity of the weakly rock mass is relatively complete and the local integrity is poor. The slightly fresh rock mass is complete or relatively complete.

Table 4 presents the elevation of the acoustic tests and the ultrasonic velocity of two boreholes in the Ronglai hydropower station. Herein, we choose mean ultrasonic velocity to calculate the GSI. Therefore, according to the Hoek-Brown criterion, the mechanical parameters of the rock mass can be determined based on the ultrasonic velocity.

Due to the convenient field conditions, a large number of shear tests have been carried out to obtain the shear strength parameters of different rocks. These measurement parameters can be used to verify the rationality of the proposed method. Therefore, we plot the evaluation results obtained by the ultrasonic velocity method, the proposed method, and the measurement values into Figure 11. The abscissa in the figure shows four rock masses in the Mamaya I hydropower station. It can be seen from Figure 11 that for four rock masses, the internal friction angle (expressed by radian) determined by the ultrasonic velocity method is larger than that obtained by the proposed method and the measurement values. The average relative error of the two methods is $11.52 \%$, and the difference is not significant. Except for rock 
TABLE 3: The ultrasonic velocity values of the rock mass in the Mamaya I hydropower station.

\begin{tabular}{|c|c|c|}
\hline Borehole no. & Elevation of rock (m) & $V_{p}(\mathrm{~m} / \mathrm{s})$ \\
\hline \multirow{5}{*}{ ZK2 } & 476.7 & 5890 \\
\hline & 473.2 & 4980 \\
\hline & 471.7 & 4200 \\
\hline & 466.7 & 5920 \\
\hline & 461.7 & 6130 \\
\hline \multirow{5}{*}{ ZK9 } & 478.5 & 5790 \\
\hline & 473.5 & 5360 \\
\hline & 468.5 & 3100 \\
\hline & 463.5 & 6100 \\
\hline & 461.5 & 5300 \\
\hline \multirow{5}{*}{ ZK17 } & 485.9 & 4690 \\
\hline & 480.9 & 6250 \\
\hline & 475.9 & 3520 \\
\hline & 470.9 & 6210 \\
\hline & 465.9 & 6130 \\
\hline \multirow{5}{*}{ ZK5 } & 477.9 & 5400 \\
\hline & 473.4 & 3960 \\
\hline & 467.9 & 5910 \\
\hline & 463.9 & 6170 \\
\hline & 461.4 & 6210 \\
\hline \multirow{5}{*}{ ZK11 } & 475.4 & 3650 \\
\hline & 470.4 & 6250 \\
\hline & 465.4 & 6060 \\
\hline & 460.4 & 5780 \\
\hline & 457.4 & 5480 \\
\hline \multirow{5}{*}{ ZK18 } & 485 & 5930 \\
\hline & 480 & 5820 \\
\hline & 477 & 2990 \\
\hline & 475 & 3770 \\
\hline & 473 & 6250 \\
\hline \multirow{5}{*}{ ZK7 } & 478.7 & 5790 \\
\hline & 473.7 & 6210 \\
\hline & 468.7 & 3680 \\
\hline & 463.7 & 5890 \\
\hline & 460.7 & 5680 \\
\hline \multirow{5}{*}{ ZK13 } & 479.1 & 5440 \\
\hline & 474.1 & 6170 \\
\hline & 469.1 & 4200 \\
\hline & 464.1 & 6090 \\
\hline & 461.1 & 6130 \\
\hline \multirow{5}{*}{ ZK20 } & 485.9 & 4690 \\
\hline & 480.9 & 6250 \\
\hline & 475.9 & 3520 \\
\hline & 470.9 & 6210 \\
\hline & 465.9 & 6130 \\
\hline
\end{tabular}

mass 1 , the measurement values are larger than those obtained by the proposed method. The average relative error of the values obtained by the proposed method and the measurement values is $5.06 \%$, and the difference is very small. Furthermore, the average relative error of the values obtained by the ultrasonic velocity method and the measurement values is $8.19 \%$, and the difference is little. On the whole, the calculated results are basically consistent with the measurement values of the rock mass in the Mamaya I hydropower station. Therefore, it is reasonable to obtain the shear strength mechanical parameters of the study area by
TABLE 4: The ultrasonic velocity values of the rock mass in the Ronglai hydropower station.

\begin{tabular}{lccc}
\hline $\begin{array}{l}\text { Borehole } \\
\text { no. }\end{array}$ & $\begin{array}{c}\text { Hole } \\
\text { depth } \\
(\mathrm{m})\end{array}$ & $\begin{array}{c}\text { Interval ultrasonic } \\
\text { velocity }(\mathrm{m} / \mathrm{s})\end{array}$ & $\begin{array}{c}\text { Mean ultrasonic } \\
\text { velocity }(\mathrm{m} / \mathrm{s})\end{array}$ \\
\hline \multirow{3}{*}{ ZK5 } & $2.6 \sim 4.0$ & $3140 \sim 3360$ & 3250 \\
& $4.0 \sim 18.0$ & $3370 \sim 5250$ & 4340 \\
& $18.0 \sim 18.8$ & $3070 \sim 3740$ & 3340 \\
\multirow{2}{*}{ ZK12 } & $18.8 \sim 59.4$ & $2970 \sim 5250$ & 4640 \\
\hline
\end{tabular}

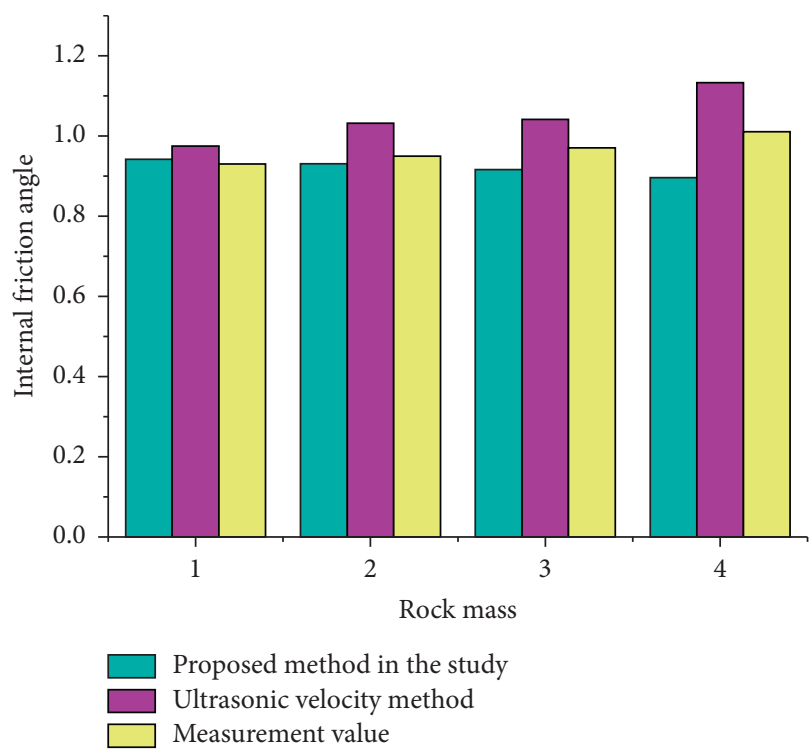

FIGURE 11: Comparison of the mechanical parameters of the rock mass from two methods and test values in the Mamaya I hydropower station.

using the proposed method in this study. The results can meet the requirements of calculation accuracy required by engineering practice in the study area, and achieve the purpose of low cost and safety.

Afterward, we plot the evaluation results obtained by the ultrasonic velocity method, the proposed method, and the measurement values into Figure 12. The abscissa in the figure shows four rock masses in the Ronglai hydropower station. For three rock masses, the internal friction angle (expressed by radian) determined by the ultrasonic velocity method is larger than that obtained by the proposed method, while it is lower than that of the measurement values. The average relative error of the two methods is $4.52 \%$, and the difference is very small. The average relative error of the values obtained by the proposed method and the measurement values is $13.85 \%$, and the difference is not significant. In addition, the average relative error of the values obtained by the ultrasonic velocity method and the measurement values is $9.80 \%$, and the difference is little. Similarly, the results show that the calculated results are basically in agreement with the measurement values of the rock mass in the Ronglai hydropower station. Therefore, the rationality of the proposed method is proved again. 


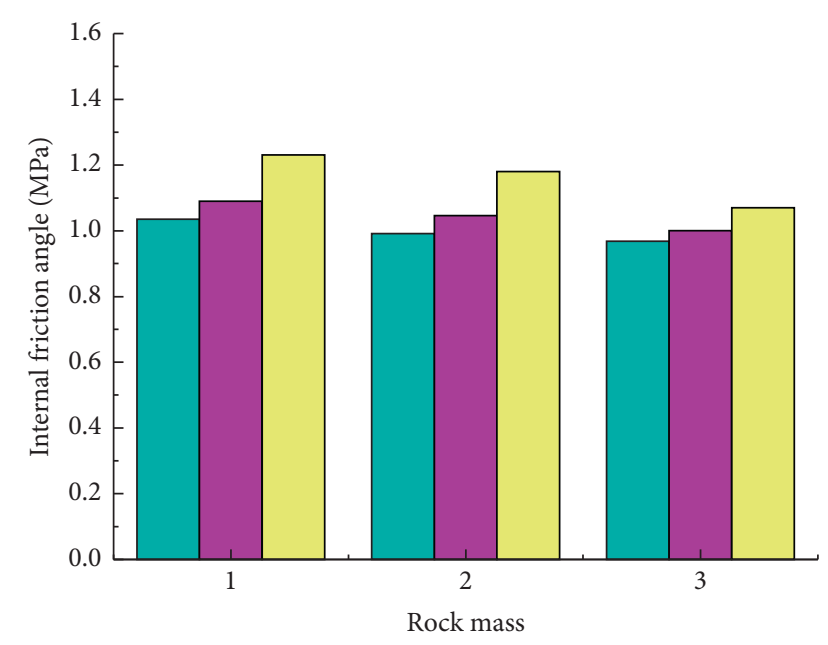

Proposed method in the study

Ultrasonic velocity method

Measurement value

FIgURE 12: Comparison of the mechanical parameters of the rock mass from two methods and test values in the Ronglai hydropower station.

\section{Discussion}

The limestone belongs to sedimentary rock. When subjected to tectonic activity and dissolution, the limestone contains a large number of defects, such as small cracks and holes, large faults, karst caves and pipelines, etc., accompanying with groundwater activities. Due to the heterogeneity and the porosity of the limestone, the internal grains pores will directly affect the physical and mechanical properties of the rock. From qualitative to quantitative characterization of pore distribution, mastering the development of pores is of great significance to analyze and evaluate the physical and mechanical properties of the rock. The compressive strength that is regarded as the basic mechanical property of the rock materials is the first factor to be considered in structural design and quality control of engineering [39]. Under the same water environment, the compressive strength shows obvious difference with a different initial porosity of the rock. However, there is little research on the mechanism of the porosity on the strength of the rock under natural and saturated conditions [40, 41]. Thus, it is necessary to explore the distribution of pores and the size of the porosity of the rock, and it has a guiding significance for the implementation of support and protection measures in rock engineering.

The limestone in this kind of stratum often presents obvious anisotropy, which is mainly reflected in the integrity of the rock mass and the groundwater. In the areas where faults, karst caves, and pipelines are developed, the integrity of the rock mass is relatively poor and the groundwater is visible. Furthermore, the grade of the surrounding rock is relatively weak, and the engineering properties of the rock mass are poor. However, in the section with only fractures and holes, the selfstability of the rock mass is relatively good, and the engineering properties of the rock mass are also relatively good, and the groundwater is generally developed.
Large geological anomalies such as faults, karst caves, and pipelines in the limestone stratum can be revealed by engineering geological mapping, geophysical prospecting, and drilling. In order to cope with this special project, many mature treatment measures have been developed. The main defects in the solution pore and crevice limestones are crystal cave and dissolution fracture. The distribution of the pores and crevices in these limestones is random. Generally, calcite is cemented or not filled, and the limestone has high hardness. This kind of limestone is widely encountered in the Mamaya I hydropower station and Ronglai hydropower station. Generally, in the thin to medium thick limestone stratum, the existence of these pores and crevices destroys the continuity and integrity of the rock mass, and changes the mechanical properties of the limestones to a certain extent.

At present, many researches have been carried out on the mechanical properties of rock or the rock mass caused by pores and crevices [42-44], but most of them are based in traditional continuum theory or discontinuous fracture mechanics. In the engineering application of the solution pore and crevice limestones, these methods often lead to excessive or conservative results. From a large number of engineering practices, it is found that some limestones in the stratum have good engineering geological properties while some limestones have poor engineering geological properties, which should be treated differently.

The main methods to determine the mechanical parameters of the rock mass include test method, numerical analysis method, displacement back analysis method, uncertainty analysis method, and empirical analysis method. Among them, the empirical analysis method is widely used in engineering practice. It is mainly based on the Hoek-Brown strength criterion and the GSI. However, the GSI depends on the quality of the rock fracture, joint surface, and the development degree of the rock mass structure. In actual engineering practice, the quantification of the GSI is often complex, with strong subjectivity, resulting in a large deviation in the mechanical parameters of the rock mass.

Based on the analysis of a large number of engineering experimental data for the solution pore and crevice limestones, combining with the Hoek-Brown criterion, this paper proposes the method for determining the rock mass mechanical parameters by substituting the porosity into the GSI. The porosity can be obtained quantitatively by the specific gravity and the dry density of the rocks, which simplify the tedious process of the GSI quantification, avoids the subjectivity, and has good reliability and suitability. Therefore, it has a certain guiding significance for engineering construction in the fields of the solution pore and crevice limestones.

\section{Conclusions}

(1) Uniaxial compression tests of the limestones are conducted. The porosity of the rock samples is determined based on the specific gravity and the dry density. The relationships between the UCS and the 
porosity of the rocks are discussed. The results illustrate that with the increase of the porosity, both the natural UCS and the saturated UCS, of these limestones decrease continuously. When the porosity is less than $1 \%$, the compressive strength decreases greatly, and it decreases slowly when the porosity is more than $5 \%$. Moreover, the relationships between the UCS and the porosity under natural and saturated conditions have good power function characteristic when $n=0.5 \% \sim 5 \%$.

(2) The method for determining mechanical parameters of the solution pore and crevice limestone was proposed based on the Hoek-Brown criterion. Firstly, the expressions of the empirical parameters for the rock mass and the porosity are established. Then depending on the Hoek-Brown criterion, the method for determining mechanical parameters, including the UCS, the TS, the $E_{m}$, the $c_{m}$ and the $\varphi_{m}$, are proposed. The proposed method simplifies the complexity of mechanical parameters by quantifying the GSI, avoids the subjectivity and uncertainty of quantifying the GSI, and has good reliability and suitability in the formation of the solution pore and crevice limestone.

(3) Based on the proposed method, the variation laws of the mechanical parameters of the limestones are analyzed. The variation of the UCS, the TS, and the $E_{m}$ present descending trends generally, and their decreasing rates with the porosity of the rock are the largest when $n=0.5 \% \sim 1 \%$. Under compression conditions, the shear strength parameters suffer different levels of degeneration as the porosity increases, but the rangeability of the $\varphi_{m}$ remains less than the $c_{m}$. Under tensile conditions, the $\varphi_{m}$ increases rapidly with the porosity, while $c_{m}$ decreases rapidly. The porosity of the rock is controlled by the TS. Furthermore, the rock is very sensitive to the tension action, so the rock shows rapid fracture instability.

\section{Data Availability}

Data used to support this study are included within the article.

\section{Conflicts of Interest}

The authors declare no conflicts of interest.

\section{Authors' Contributions}

Zheng $\mathrm{Hu}$ and Tao Wen contributed equally to the article. Tao Wen, Zheng $\mathrm{Hu}$, and Kexun Zheng conceived the study design; Tao Wen and Zheng $\mathrm{Hu}$ analyzed the data and wrote the manuscript; Yankun Wang helped to edit the manuscript.

\section{Acknowledgments}

This work was funded by the Key National Natural Science Foundation of China (No. 42002268), Open Foundation of
Hubei Key Laboratory of Blasting Engineering (HKLBEF20201 2), Open Foundation of Engineering Research Center of Rock-Soil Drilling and Excavation and Protection, Ministry of Education (202006), Open Fund of Hubei Cooperative Innovation Center of Unconventional Oil and Gas (Yangtze University) (UOG2020-11), and Open Foundation of Top Disciplines in Yangtze University.

\section{References}

[1] T. Wen, H. M. Tang, Y. K. Wang, and J. W. Ma, "Evaluation of methods for determining rock brittleness under compression," Journal of Natural Gas Science and Engineering, vol. 2020, Article ID 103321, 2020.

[2] J. Zheng, Y. Zhao, Q. Lü, J. Deng, X. Pan, and Y. Li, “A discussion on the adjustment parameters of the Slope Mass Rating (SMR) system for rock slopes," Engineering Geology, vol. 206, pp. 42-49, 2016.

[3] H. Munoz, A. Taheri, and E. K. Chanda, "Rock drilling performance evaluation by an energy dissipation based rock brittleness index," Rock Mechanics and Rock Engineering, vol. 49, no. 8, pp. 3343-3355, 2016.

[4] J. Tang, C. Ehlig-Economides, B. Fan, B. Cai, and W. Mao, "A microseismic-based fracture properties characterization and visualization model for the selection of infill wells in shale reservoirs," Journal of Natural Gas Science and Engineering, vol. 67, pp. 147-159, 2019.

[5] B. Tarasov and Y. Potvin, "Universal criteria for rock brittleness estimation under triaxial compression," International Journal of Rock Mechanics and Mining Sciences, vol. 59, pp. 57-69, 2013.

[6] T. Wen, H. M. Tang, L. Huang, Y. K. Wang, and J. W. Ma, "Energy evolution: a new perspective on the failure mechanism of purplish-red mudstones from the three gorges reservoir area, China," Engineering Geology, vol. 2020, Article ID 105350, 2020.

[7] M. Bahaaddini, G. Sharrock, and B. K. Hebblewhite, "Numerical investigation of the effect of joint geometrical parameters on the mechanical properties of a non-persistent jointed rock mass under uniaxial compression," Computers and Geotechnics, vol. 49, pp. 206-225, 2013.

[8] E. Hoek and M. S. Diederichs, "Empirical estimation of rock mass modulus," International Journal of Rock Mechanics and Mining Sciences, vol. 43, no. 2, pp. 203-215, 2006.

[9] E. Hoek, C. Carranza-Torres, and B. Corkum, "Hoek-Brown failure criterion-2002 Edition," in Proceedings of 5th North American Rock Mechanics Symposium, pp. 267-273, Toronto, Canada, July 2002.

[10] M. Singh and B. Singh, "Modified Mohr-Coulomb criterion for non-linear triaxial and polyaxial strength of jointed rocks," International Journal of Rock Mechanics and Mining Sciences, vol. 51, pp. 43-52, 2012.

[11] M. Singh, A. Raj, and B. Singh, "Modified Mohr-Coulomb criterion for non-linear triaxial and polyaxial strength of intact rocks," International Journal of Rock Mechanics and Mining Sciences, vol. 48, no. 4, pp. 546-555, 2011.

[12] R. Huang, W. Liu, J. Zhou, and X. Pei, "Experimental field study of movement charateristics of rock blocks falling down a slope," Journal of Earth Science, vol. 21, no. 3, pp. 330-339, 2010.

[13] N. Gatelier, F. Pellet, and B. Loret, "Mechanical damage of an anisotropic porous rock in cyclic triaxial tests," International 
Journal of Rock Mechanics and Mining Sciences, vol. 39, no. 3, pp. 335-354, 2002.

[14] R. Q. Huang, X. N. Wang, and L. S. Chan, "Triaxial unloading test of rocks and its implication for rock burst," Bulletin of Engineering Geology and the Environment, vol. 60, no. 1, pp. 37-41, 2001.

[15] H. Q. Xie and C. H. He, "Study of the unloading characteristics of a rock mass using the triaxial test and damage mechanics," International Journal of Rock Mechanics and Mining Sciences, vol. 41, pp. 74-80, 2004.

[16] M. Cai, P. K. Kaiser, Y. Tasaka, and M. Minami, "Determination of residual strength parameters of jointed rock masses using the GSI system," International Journal of Rock Mechanics and Mining Sciences, vol. 44, no. 2, pp. 247-265, 2007.

[17] M. Cai, P. K. Kaiser, H. Uno, Y. Tasaka, and M. Minami, "Estimation of rock mass deformation modulus and strength of jointed hard rock masses using the GSI system," International Journal of Rock Mechanics and Mining Sciences, vol. 41, no. 1, pp. 3-19, 2004.

[18] M. Cai, H. Morioka, P. K. Kaiser et al., "Back-analysis of rock mass strength parameters using AE monitoring data," International Journal of Rock Mechanics and Mining Sciences, vol. 44, no. 4, pp. 538-549, 2007.

[19] X.-T. Feng, Z. Zhang, and Q. Sheng, "Estimating mechanical rock mass parameters relating to the Three Gorges Project permanent shiplock using an intelligent displacement back analysis method," International Journal of Rock Mechanics and Mining Sciences, vol. 37, no. 7, pp. 1039-1054, 2000.

[20] K. Tajduś, "New method for determining the elastic parameters of rock mass layers in the region of underground mining influence," International Journal of Rock Mechanics and Mininig Sciences, vol. 46, no. 8, pp. 1296-1305, 2009.

[21] R. Yoshinaka, M. Osada, H. Park, T. Sasaki, and K. Sasaki, "Practical determination of mechanical design parameters of intact rock considering scale effect," Engineering Geology, vol. 96, no. 3-4, pp. 173-186, 2008.

[22] G. F. Andriani and M. Parise, "Applying rock mass classifications to carbonate rocks for engineering purposes with a new approach using the rock engineering system," Journal of Rock Mechanics and Geotechnical Engineering, vol. 9, no. 2, pp. 364-369, 2017.

[23] Z. Şen and B. H. Sadagah, "Modified rock mass classification system by continuous rating," Engineering Geology, vol. 67, no. 3, pp. 269-280, 2003.

[24] Z. T. Bieniawski, "Engineering classification of joint rock masses," The Civil Engineer In South Africa, vol. 15, no. 12, pp. 335-343, 1973.

[25] Z. T. Bieniawski, "Geomechanics classification of rock masses and its application in tunneling," in Proceedings of the $3 \mathrm{rd}$ International Congress on Rock Mechanics, pp. 27-32, Denver, CO, USA, September 1974.

[26] M. Romana, R. Tomás, and J. B. Serón, "Slope mass rating (SMR) geomechanics classification: thirty years review," in Proceedings of the 13th ISRM International Congress of Rock Mechanics, International Society for Rock Mechanics, Montréal, Canada, September 2015.

[27] T. Wen, H. Tang, and Y. Wang, "Brittleness evaluation based on the energy evolution throughout the failure process of rocks," Journal of Petroleum Science and Engineering, vol. 194, Article ID 107361, 2020.

[28] Z. W. Ding, X. F. Li, Q. B. Tang, and J. D. Jia, "Study on correlation between fractal characteristics of pore distribution and strength of sandstone particles," Chinese Journal of Rock Mechanics and Engineering, vol. 39, pp. 1-10, 2020.
[29] M. Cai, "Practical estimates of tensile strength and HoekBrown strength parameter $\mathrm{m}$ i of brittle rocks," Rock Mechanics and Rock Engineering, vol. 43, no. 2, pp. 167-184, 2010.

[30] E. Hoek and E. T. Brown, "Empirical strength criterion for rock masses," Journal of Geotechnical and Geoenvironmental Engineering, vol. 106, pp. 1013-1035, 1980.

[31] R. Ajalloeian and M. Mohammadi, "Estimation of limestone rock mass deformation modulus using empirical equations," Bulletin of Engineering Geology and the Environment, vol. 73, no. 2, pp. 541-550, 2014.

[32] J. Peng, G. Rong, M. Cai, X. Wang, and C. Zhou, "An empirical failure criterion for intact rocks," Rock Mechanics and Rock Engineering, vol. 47, no. 2, pp. 347-356, 2014.

[33] L. R. Richards and S. A. L. Read, "A comparison of methods for determining mi, the Hoek-Brown parameter for intact rock material," in Proceedings of the 45th US Rock Mechanics/ Geomechanics Symposium, American Rock Mechanics Association, San Francisco, CA, USA, June 2011.

[34] E. Hoek and E. T. Brown, "The Hoek-Brown failure criteriona 1988 update," in Proceedings of the 15th Canadian Rock Mechanics Symposium, Dept. Civil Engineering, University of Toronto, Toronto, Canada, July 1988.

[35] J. L. Justo, J.-C. García-Núñez, M. Vázquez-Boza, E. Justo, P. Durand, and J. M. Azañón, "Design of raft foundations for high-rise buildings on jointed rock," Rock Mechanics and Rock Engineering, vol. 47, no. 4, pp. 1277-1290, 2014.

[36] M. Hashemi, S. Moghaddas, and R. Ajalloeian, "Application of rock mass characterization for determining the mechanical properties of rock mass: a comparative study," Rock Mechanics and Rock Engineering, vol. 43, no. 3, pp. 305-320, 2010.

[37] K. Z. Xia, C. X. Chen, X. M. Liu, Y. Zheng, and Y. C. Zhou, "Estimation of rock mass mechanical parameters based on ultrasonic velocity of rock mass and Hoek-Brown criterion and its application of engineering," Chinese Journal of Rock Mechanics and Engineering, vol. 32, no. 7, pp. 1458-1466, 2013.

[38] Y. Song and G. Ju, "Determination of rock mass shear strength based on in-situ tests and codes and comparison with estimation by Hoek-Brown criterion," Chinese Journal of Rock Mechanics and Engineering, vol. 31, no. 5, pp. 1000-1006, 2012.

[39] J. R. Shen, S. G. Li, and Q. J. Xu, "Mechanism of Saturation and Porosity on compressive strength of concrete," Journal of Building Materials, pp. 1-12, 2020.

[40] Z. M. Huang, G. Y. Cao, H. Cheng, M. Y. Ma, and Y. S. Huang, "Correlation analysis of porosity and uniaxial compressive strength of jurassic frozen sandstone," Safety in Coal Mines, vol. 51, no. 6, pp. 227-231, 2020.

[41] Y. Tian, "Triaxial compression strength and fracture development of shale with different initial porosity," Mineral Engineering Research, vol. 34, no. 4, pp. 40-43, 2019.

[42] M. R. Du, H. W. Jing, H. J. Su, and T. T. Zhu, "Effects of holes geometrical shape on strength and failure characteristics of a sandstone sample containing a single hole," Engineering Mechanics, vol. 33, no. 7, pp. 190-196, 2016.

[43] X. L. Liu, S. J. Wang, E. Z. Wang, J. X. Wang, and B. Hu, "Evolutionary rules of flaws in rock subjected to uniaxial compression and rock strength," Chinese Journal of Rock Mechanics and Engineering, vol. 6, pp. 1195-1201, 2008.

[44] S. Q. Yang, X. R. Liu, and Y. S. Li, "Experimental analysis of mechanical behavior of sandstone containing hole and fissure under uniaxial compression," Chinese Journal of Rock Mechanics and Engineering, vol. 31, no. S2, pp. 3539-3546, 2012. 